\title{
Prevalencia de trastornos depresivos e impedimento asociado en adolescentes de sexo femenino
}

\author{
FÉLIX COVA S. ${ }^{1 a}$, PAULINA RINCÓN G. ${ }^{1 a}$ MARIO VALDIVIA P. ${ }^{2 b}$, ROBERTO MELIPILLÁN A. ${ }^{3 c}$ \\ 1. Departamento de Psicología, Facultad de Ciencias Sociales, Universidad de Concepción. \\ 2. Departamento de Psiquiatría, Facultad de Medicina, Universidad de Concepción. \\ 3. Universidad San Sebastián, Concepción. \\ a. Doctor en Psicología, Psicólogo. \\ b. Médico Cirujano, Psiquiatra Infantil. \\ c. Magíster, Psicólogo.
}

\begin{abstract}
Depressive disorders prevalence and associated impediments in female adolescents

Background: From early adolescence, depressive disorders experience a significant increase, especially in female population, showing not only immediate adverse consequences, but also at later stages in adult development. Although its importance, there are few studies regarding the prevalence of these disorders in chilean adolescents. Methods: Estimation of prevalence of major depression and dysthymic disorders in 172 students from first year of secondary education. The computerized interview DISC-IV obtained a diagnosis based on DSM-IV criteria. Results: $10 \%$ of the population showed a depressive episode in the last year and more than $5 \%$ in the last month. The incidence of dysthymic disorder was low $(1.2 \%)$. The incidence of major depressive subthreshold in the last year was $11.6 \%$ and $8.5 \%$ for subthreshold dysthymic disorder. Conclusions: Most of female with depressive disorders and subthreshold disorders presented impediments related to symptoms experienced. Although these high rates might suggest problems in the conceptualization of depressive disorders, they constitute the result of strict DSM-IV criteria application, including clinical significant criteria. These results are indicative of the need to pay attention to emotional wellbeing of female adolescents.

(Key words: adolescence, epidemiology, depressive disorder).

Rev Chil Pediatr 2008; 79 (6): 607-613
\end{abstract}

\section{RESUMEN}

Introducción: Los trastornos depresivos se incrementan de modo notorio a partir del inicio de la adolescencia, especialmente en las mujeres, con efectos adversos tanto inmediatos como en el desarrollo posterior. Pese a su importancia, en Chile existen pocos estudios de la prevalencia de estos trastornos en

Trabajo recibido el 16 de septiembre de 2008, devuelto para publicación el 22 de octubre de 2008, segunda versión el 05 de noviembre de 2008, aceptado para publicación el 19 de noviembre de 2008.

Financiamiento Proyecto: Fondecyt 11060059

Correspondencia a:

Félix Cova S.

E-mail: fecova@udec.cl 
adolescentes. Método: Se estimó la prevalencia de último mes y de último año de depresión mayor y de distimia, en una muestra de 172 estudiantes mujeres de primero medio de liceos de Concepción. Se utilizó la entrevista digitalizada DISC-IV que permite obtener diagnóstico según criterios DSM-IV. Resultados: Casi un $10 \%$ de las niñas presentaron un episodio depresivo mayor en el último año y poco más de un 5,0\% en el último mes; la prevalencia de trastorno distímico fue muy baja, 1,2\%. La prevalencia de último año de episodio depresivo mayor subumbral fue de $11,6 \%$ y la de distimia de $8,5 \%$. En la mayoría de las niñas con trastornos depresivos y con trastornos subumbrales se observó presencia de malestar o impedimento asociado a los síntomas experimentados. Conclusiones: Si bien estas tasas tan elevadas pueden estar sugiriendo dificultades en la conceptualización de los trastornos depresivos, son el resultado de la aplicación estricta de los criterios DSM-IV, incluyendo el criterio de significación clínica, e indican la necesidad de poner atención en el bienestar emocional de las adolescentes.

(Palabras clave: Adolescencia, epidemiología, trastornos depresivos).

Rev Chil Pediatr 2008; 79 (6): 607-613

\section{Introducción}

La conceptualización de los trastornos depresivos en la adolescencia, en particular de los trastornos depresivos "unipolares", que son el objeto de atención del presente artículo, ha sido un tema complejo y controversial. Dos aspectos centrales de las controversias han sido: a) cómo diferenciar en los adolescentes estados depresivos con significación clínica efectiva, que puedan ser conceptualizados como un trastorno psicopatológico propiamente tal, de estados de ánimo negativos transitorios y relacionados con los estresores psicosociales habituales de este momento del ciclo vital ${ }^{1}$; y b) hasta dónde son aplicables a esta etapa de la vida los criterios aceptados para diagnosticar trastornos depresivos, generados originalmente a partir de población adulta ${ }^{2}$.

Pese a que ambos temas continúan sin ser resueltos claramente, los investigadores y profesionales del ámbito de la salud mental han optado mayoritariamente por identificar los trastornos depresivos en niños y adolescentes siguiendo los criterios establecidos por la Asociación Psiquiátrica Americana -en particular, los expresados en la cuarta edición del $\mathrm{DSM}^{3}$ - . Estos criterios son, excepto aspectos específicos, los mismos para niños, adolescentes y adultos $^{2}$. La investigación basada en estos criterios ha establecido que la prevalencia de trastornos depresivos se incrementa de modo notorio a partir del inicio de la adolescencia, pasando a constituir uno de los trastornos psicopatológicos más frecuentes en los adolescentes, con efec- tos adversos tanto inmediatos como en el desarrollo posterior de los adolescentes ${ }^{4}$.

La mayoría de las investigaciones sitúan la prevalencia anual del trastorno depresivo mayor entre 4 a $7 \%$ en adolescentes ${ }^{5}$. Las estimaciones de prevalencia de distimia son semejantes a las del trastorno depresivo mayor aunque se trata de un trastorno menos estudiado ${ }^{4}$. Estas cifras corresponden a estudios que se han realizado utilizando instrumentos especialmente construidos para evaluar, del modo más confiable posible, el cumplimiento de los criterios diagnósticos DSM o CIE en muestras poblacionales o, en su defecto, en población escolar.

La investigación de los trastornos mentales basada en criterios DSM-IV, incluyendo con especial relevancia la centrada en los trastornos depresivos, está examinando cada vez más la significación clínica de los síntomas y/o impedimento como una variable complementaria al diagnóstico ${ }^{6}$. Estudios con adolescentes han mostrado que jóvenes con diagnósticos subumbrales de trastornos depresivos pueden tener consecuencias adversas en sus vida y niveles de impedimento equivalentes a las de quienes reúnen todos los criterios diagnósticos ${ }^{7,8}$; por el contrario, también se ha observado que cumplir los criterios diagnósticos de un trastorno no es por sí mismo indicador de la presencia de un trastorno incapacitante ni de necesidad de tratamiento ${ }^{9,10}$.

En nuestro país, existen muy pocos estudios de los trastornos depresivos en niños y adolescentes que se hayan realizado en muestras no clínicas. Un estudio de seguimiento en escola- 
res de Santiago de $1^{\circ}$ y $6^{\circ}$ básico con criterios CIE-10, mostró que quienes recibían el diagnóstico de trastorno depresivo eran los que tenían más discapacidad asociada -del conjunto de trastornos evaluados- ${ }^{6}$.

Sin emplear instrumentos que permitan evaluar trastornos, sino exclusivamente sintomatología, sí se han realizado estudios de prevalencia de sintomatología depresiva en escolares. Estos estudios han utilizado cuestionarios que permiten cuantificar frecuencia y/o intensidad de sintomatología depresiva, pero no hacer diagnósticos de trastorno propiamente según criterios DSM-IV o CIE-10. Los resultados obtenidos han indicado la presencia de significativos niveles de sintomatología depresiva en los grupos estudiados ${ }^{11,12}$.

En una muestra representativa de adolescentes de enseñanza media, empleando el puntaje de corte original del Inventario de Depresión de Beck II para sintomatología depresiva moderada o grave, se encontró que poco más del 30\% de los adolescentes calificaba en estas categorías ${ }^{13}$. La misma cifra de sintomatología depresiva fue obtenida en la Encuesta de Salud Escolar del año 2004 aplicada en cuatro regiones de Chile a una muestra amplia de adolescentes entre 13 y 15 años ${ }^{14}$. La comparabilidad de estos resultados con los de estudios internacionales es limitada, pero sugieren una mayor presencia de autoinforme de sintomatología depresiva en los adolescentes chilenos, sin que exista claridad respecto de cómo explicar estos resultados ${ }^{13}$.

A partir de la adolescencia los trastornos depresivos aumentan en ambos géneros, pero lo hacen de modo mucho más acentuado en las adolescentes ${ }^{15}$. Entre los 13 y 14 años es ya observable que las niñas presentan tasas de depresión más elevadas que los niños ${ }^{16}$, estas diferencias de género se continúan acentuando entre los 15 y 18 años para luego estabilizarse ${ }^{17}$.

La presente investigación tiene como objetivo estimar las tasas de depresión (episodio depresivo mayor y distimia) que son observables en adolescentes mujeres estudiantes de primer año de enseñanza media al aplicar un instrumento especialmente diseñado para hacer diagnóstico según criterios DSM-IV. Dada la relevancia indicada de los trastornos subum- brales se hace también una estimación de la prevalencia de éstos. Se indican y comparan los resultados obtenidos incluyendo y excluyendo la consideración de los niveles de malestar o impedimento asociados a la presencia de síntomas.

\section{Métodos}

Ciento ochenta y ocho niñas de primero medio, correspondientes al total de niñas de primero medio de tres establecimientos educacionales ( 7 cursos en total), fueron las participantes consideradas originalmente en el estudio; la muestra total efectivamente obtenida fue de 172 . De éstas, 39 pertenecían a un colegio particular subvencionado, 54, a una escuelaliceo mixta municipal y 79, a una escuela liceo sólo de niñas, también municipal. Todos los establecimientos correspondían a unidades educativas que atienden a población de sectores bajos y medio bajos, pero no de extrema pobreza (Índices de Vulnerabilidad entre 42,3\% y $54,9 \%$, de acuerdo a la estadísticas de la Junta Nacional de Auxilio Escolar y Becas $)^{18}$. El rango de edad osciló entre 13 a 17 años, estando más del $90 \%$ de las niñas entre 14 y 15 años; un $2,7 \%$ tenían 13 años.

A todas las niñas se les solicitó su consentimiento informado para participar del estudio. Los padres también fueron informados de la investigación. No existió rechazo a participar de la investigación. A las niñas (y a sus padres) que aparecieron con diagnóstico positivo de trastorno depresivo se las contactó en forma escrita y confidencial indicando la posible existencia de un problema que podría requerir atención profesional y se les ofreció apoyo para obtener ésta.

Para el estudio se empleó el módulo referido a trastornos del estado de ánimo de la versión española de la entrevista estructurada National Institute of Mental Health Diagnostic Interview Schedule for Children Version IV (NIMH DISC-IV), en su modalidad digital ${ }^{19}$. Se empleó la versión que es respondida por los propios adolescentes.

El NIMH-DISC-IV es una entrevista altamente estructurada, diseñada para ser adminis- 
trada por entrevistadores legos, que permite obtener diagnóstico de acuerdo a los criterios DSM-IV $^{20}$. Se trata de una de las entrevistas más empleadas actualmente en la investigación internacional actualmente en epidemiología psiquiátrica infantojuvenil. Se han obtenido niveles comparables de confiabilidad test-retest entre la versión original en inglés y la española ${ }^{19}$. Esta entrevista incluye la consideración de la significación clínica de los síntomas (malestar asociado o impedimento a su presencia), pero no utiliza la significación clínica como criterio diagnóstico (esto es, se asigna diagnóstico si se cumplen los criterios diagnósticos sin considerar la significación clínica de los síntomas, que viene a ser considerada así otro eje o dimensión). La significación clínica es evaluada en función del informe de malestar o de impedimento en distintos ámbitos de desenvolvimiento, asociados a la presencia de síntomas (relaciones con cuidadores o familiares, participación en actividades familiares y con amigos, adaptación escolar y relaciones con profesores, distrés atribuible a los síntomas). Para operacionalizar la significación clínica, en el presente estudio se utilizó como indicador de ésta la presencia de malestar o impedimento interme- dio o severo en al menos un área de funcionamiento, asociada a la presencia de los síntomas, siguiendo los criterios empleados en otras investigaciones $^{21}$. A fines de un análisis de la prevalencia con un criterio más estricto de significación clínica ("significación clínica acentuada") también se estimó la significación clínica dada por la presencia de al menos un área de funcionamiento con malestar o impedimento severo, o de al menos dos con malestar o impedimento intermedio $\mathrm{y} / \mathrm{o}$ severo.

\section{Resultados}

La tabla 1 muestra las tasas de prevalencia de último mes y de último año del trastorno depresivo mayor. Se indica además, la tasa de prevalencia del trastorno depresivo mayor con significación clínica y con significación clínica "acentuada". En la tabla 2 se indican las tasas de prevalencia del trastorno distímico. Los algoritmos diagnósticos usados en el DISC-IV excluyen la posibilidad de diagnosticar depresión doble, dado que el diagnóstico de episodio depresivo mayor excluye la posibilidad de diagnóstico de distimia ${ }^{20}$.

Tabla 1. Prevalencia de trastorno depresivo mayor

\begin{tabular}{llllll}
\hline $\begin{array}{l}\text { Prevalencia último } \\
\text { año }\end{array}$ & $\begin{array}{l}\text { Prevalencia último } \\
\text { año con significa- } \\
\text { ción clínica }\end{array}$ & $\begin{array}{l}\text { Prevalencia último } \\
\text { año con significa- } \\
\text { ción clínica } \\
\text { acentuada }\end{array}$ & $\begin{array}{l}\text { Prevalencia último } \\
\text { mes }\end{array}$ & $\begin{array}{l}\text { Prevalencia último } \\
\text { mes con significa- } \\
\text { ción clínica }\end{array}$ & $\begin{array}{l}\text { Prevalencia último } \\
\text { mes con significa- } \\
\text { ción clínica } \\
\text { acentuada }\end{array}$ \\
\hline $9,3 \%$ & $9,3 \%$ & $8,7 \%$ & $5,2 \%$ & $5,2 \%$ & $4,7 \%$ \\
\hline
\end{tabular}

Tabla 2. Prevalencia de trastorno distímico

\begin{tabular}{lll}
\hline Prevalencia último año & $\begin{array}{l}\text { Prevalencia último año con significación } \\
\text { clínica }\end{array}$ & $\begin{array}{l}\text { Prevalencia último año con significación } \\
\text { clínica acentuada }\end{array}$ \\
\hline $1,2 \%$ & $1,2 \%$ & $1,2 \%$ \\
\hline
\end{tabular}

Tabla 3. Prevalencias de trastorno depresivo mayor y de trastornos distímicos subumbrales

\begin{tabular}{|c|c|c|c|c|c|}
\hline \multicolumn{3}{|c|}{ Trastorno depresivo mayor subumbral } & \multicolumn{3}{|c|}{ Trastorno distímico subumbral } \\
\hline $\begin{array}{l}\text { Prevalencia último } \\
\text { año }\end{array}$ & $\begin{array}{l}\text { Prevalencia último } \\
\text { año con significa- } \\
\text { ción clínica }\end{array}$ & $\begin{array}{l}\text { Prevalencia último } \\
\text { año con significa- } \\
\text { ción clínica } \\
\text { acentuada }\end{array}$ & $\begin{array}{l}\text { Prevalencia último } \\
\text { año }\end{array}$ & $\begin{array}{l}\text { Prevalencia último } \\
\text { año con significa- } \\
\text { ción clínica }\end{array}$ & $\begin{array}{l}\text { Prevalencia último } \\
\text { año con significa- } \\
\text { ción clínica } \\
\text { acentuada }\end{array}$ \\
\hline $11,6 \%$ & $11,0 \%$ & $8,7 \%$ & $8,5 \%$ & $8,5 \%$ & $8,5 \%$ \\
\hline
\end{tabular}


La tabla 3 indica las tasas de prevalencia de último mes y de último año de episodio depresivo mayor y de distimia subumbral, esto es, existe sintomatología, pero no se satisfacen todos los criterios DSM-IV para el diagnóstico propiamente de cada trastorno respectivo. No existe exclusión entre los síntomas de depresión mayor y de distimia.

\section{Discusión}

De acuerdo a los algoritmos de análisis del NIMH DISC-IV, fundamentados a su vez en los criterios DSM-IV, casi un $10 \%$ de las niñas de la muestra estudiada presentó un episodio depresivo mayor en el último año y poco más de un $5 \%$ estaría presentando o habría presentado un episodio depresivo en el último mes. En todos los casos, la presencia de los síntomas tuvo alguna significación clínica en términos de generar malestar o impedimento en el funcionamiento social en los ámbitos evaluados; en más de un $90 \%$ de los casos, esta significación clínica fue acusada en términos de implicar al menos dos áreas afectadas o una severamente. La prevalencia de trastorno distímico observada fue muy baja, $1,2 \%$, y no presentó variaciones en función de la significación clínica de los síntomas. La exclusión de la depresión doble como posibilidad diagnóstica es un factor que puede influir en acentuar la baja tasa de trastorno distímico.

Tanto las tasas de prevalencia de un año de trastornos depresivos mayores subumbrales como de trastorno distímico subumbral que se observaron fueron elevadas $(11,6 \%$ y $8,5 \%$, respectivamente). La aplicación de criterios de significación clínica no disminuyen de modo relevante estas tasas de trastornos subumbrales. De hecho, la prevalencia de distimia subumbral no es afectada por la significación clínica y la presencia de trastorno depresivo mayor subumbral sólo experimenta una reducción a una prevalencia de $8,7 \%$ cuando se utiliza el criterio de significación clínica acusada.

Dado que no existen en Chile estudios que usen criterios diagnósticos DSM-IV a través de una evaluación altamente estructurada como es el NIHM DISC-IV, es difícil comparar los resultados obtenidos. Se trata, además, de una población bien específica: niñas de 14-15 años, estudiantes de primero medio, provenientes de hogares con condición socioeconómica baja (pero no extrema) o media baja. La metodología seguida se basa en el informe de síntomas exclusivamente por parte de las niñas, a diferencia de otros estudios que utilizan a los padres como fuente de información, o a los niños y a los padres.

Pese a esta dificultad para hacer comparaciones, las cifras tienden a ser más altas que las observadas en otras sociedades. El reciente estudio de González-Tejera et al $^{22}$ obtuvo, en una muestra nacional de niñas portorriqueñas de 11 a 17 años (donde también se empleó el DISC-IV en su versión de autoinforme), una tasa de prevalencia de un año de trastorno depresivo mayor de 7,23\% y de trastorno depresivo mayor subumbral de 5,95\%. Estudios de prevalencia de sintomatología depresiva también han mostrado un mayor nivel de informes de síntomas en los adolescentes chilenos ${ }^{23}$.

Con esas consideraciones, los resultados indican que ya en adolescentes chilenas de 15 años las cifras de trastornos depresivos son relevantes al aplicarse estrictamente criterios DSM-IV. Aun cuando el uso de estos criterios de modo estricto podría estar generando una sobreestimación de la tasa de trastorno depresivo que es efectivamente relevante desde el punto de vista clínico ${ }^{24}$, debe tenerse presente que los resultados obtenidos muestran que con altísima frecuencia los diagnósticos aparecen asociados a la presencia de malestar o impedimento, incluso cuando éste es evaluado con criterios más estrictos. Una explicación posible es que en nuestra cultura exista una tendencia mayor a la aquiescencia a la hora de responder autoinformes de síntomas y al valorar la significación de éstos, pero ello no ha sido evaluado.

Es importante destacar que no existe evidencia que las entrevistas estructuradas aplicadas por entrevistadores legos sobreestimen la presencia de síntomas respecto a las entrevistas clínicas, más bien la evidencia existente es la contraria ${ }^{25,26}$. De allí que la discusión actual respecto de las elevadas tasas de trastornos que ofrecen los estudios epidemiológicos esté centrada en los criterios diagnósticos mismos, 
más que en las supuestas sobreestimaciones de las entrevistas diagnósticas empleadas ${ }^{24}$.

Las cifras de trastornos subumbrales obtenidas son elevadas, tanto para la depresión mayor como para la distimia. Es de alta relevancia que el cumplimiento o no de los criterios diagnósticos no muestra ninguna relación con los grados de malestar o impedimento que generan los síntomas. Esto implica que cumplir cabalmente con los criterios diagnósticos de depresión mayor o distimia o presentar el trastorno en un grado subumbral no hace diferencia respecto del grado de malestar o impedimento que las adolescentes atribuyen a los síntomas presentados. Ello pone un signo de interrogación respecto de la validez de los criterios para diferenciar presencia y no presencia de trastorno depresivo, fenómeno que ya ha sido observado por otros investigadores ${ }^{8,22}$.

La investigación internacional es consistente en demostrar el sostenido incremento que tiene la prevalencia de sintomatología y trastornos depresivos en mujeres luego de la pubertad. Los datos obtenidos corroboran la relevancia del fenómeno incluso en niñas que están en la fase temprana de la adolescencia y obligan a generar estrategias pertinentes para esta realidad. Dada la naturaleza masiva del fenómeno y la probable incidencia de un conjunto de factores socioculturales, no parece apropiado que dichas estrategias se focalicen en medicalizar el malestar adolescente sino en fortalecer las capacidades de los adolescentes para abordar las dificultades de la vida y en mejorar los sistemas de soporte a que acceden.

Es ampliamente discutido qué significa, con los conceptos e instrumentos empleados hoy, satisfacer los criterios diagnósticos de un trastorno determinado ${ }^{27,28}$. Un aspecto importante al respecto es que si bien existe cierto consenso en la relevancia de acompañar los diagnósticos con una evaluación de la significación clínica de los síntomas, no se ha desarrollado una metodología para hacerlo suficientemente elaborada. De hecho, la metodología que emplea el DISCIV al respecto, y que fue la utilizada en la presente investigación, todavía no recibe un estudio psicométrico cuidadoso de sus características.

Aparte de esta última limitación, el presente estudio presenta otras limitaciones, en particular, las relativas a lo pequeño del tamaño muestral, que impiden generalizar los hallazgos. Investigaciones con muestras más amplias permitirán tener más claridad respecto de la validez de los resultados obtenidos. Sin embargo, el desafío mayor de la investigación futura parece estar en el plano conceptual, en particular, en deslindar adecuadamente las implicaciones del concepto de trastorno.

\section{Referencias}

1.- Kessler RC, Avenevoli S, Ries Merikangas K: Mood disorders in Children and Adolescent: An epidemiological perspective. Biol Psychiatry 2001; 49: 100214.

2.- Avenevoli S, Knight E, Kessler R, Merikangas KR: Epidemiology of depression in children and adolescent: A developmental Psychopathological Approach. En Abela JRZ, Hankin BL, editores. New York: Guildford Press 2008; 6-32.

3.- Asociación Psiquiátrica Americana: Manual Diagnóstico y Estadístico de los Trastornos Mentales. Barcelona: Masson; 1994.

4.- Harrington RC: Affective disorders. En Rutter, M., Taylor, E., editores. Oxford: Blackwell Publishing Science; 2002. p. 463-85.

5.- Short A, Spence S: Risk and protective factors for depression in youth. Behaviour Change 2006; 23: 130 .

6.- De la Barra F, Toledo V, Rodríguez J: Revista Chilena de Neuro-Psiquiatría 2004; 42: 259-72.

7.- Kessler RC, Zhao S, Blazer DG, Swartz M: Prevalence, correlates, and course of minor depression and major depression in the National Comorbidity Survey. Journal of Affective Disorders 1997; 45: 19-30.

8.- Pickles A, Rowe R, Simonoff E, Foley D, Rutter M, Silberg $J$ : Child psychiatric symptoms and psychosocial impairment: relationship and prognostic significance. British Journal of Psychiatry 2001; 179: 230-5.

9.- Spitzer R, Wakefield J: DSM-IV diagnostic criterion for clinical significance: Does it helpe solve the false positives problem? Am J Psychiatry 1999; 156: 185664.

10.- Kendell R, Jablensky A: Distinguishing between the validity and utility of psychiatric diagnoses. Am J Psychiatry 2003; 160: 4-12.

11.- Cumsille P, Martínez M: Síntomas de depresión en estudiantes de enseñanza media de Santiago. Rev Chil Pediatr 1997; 68: 74-7.

12.- Salvo L: Ideación e intento de suicidio en adolescentes de enseñanza media en la comuna de Concepción. Tesis para optar a la especialización en psiquiatría. Concepción: Facultad de Medicina, Universidad de Concepción; 1995.

13.- Cova F, Melipillán R, Valdivia M, Bravo E, Valenzuela 
$B$ : Sintomatología depresiva y ansiosa en estudiantes de enseñanza media. Revista Chilena de Pediatría 2007; 78: 151-9.

14.- Ministerio de Salud Chile: Encuesta Mundial de Salud Escolar Chile 2004-2005. Disponible en http:// epi.minsal.cl/epi/html/invest/EMSE/INFORME\%20 EMSE\%202004-2005\%20final.pdf . Obtenido 01/11/ 2008.

15.- Hankin BL, Wetter E, Cheely C: A Sex differences in child and adolescent depression: A developmental Psychopathological Approach. En Abela, J.R.Z., Hankin, B.L. editores. New York: Guildford Press 2008; 377-414.

16.- Wade T, Cairney J, Pevalin D: Emergence of gender differences in depression during adolescence: National panel results from three countries. J Am Acad Child Adolesc Psychiatry 2002; 41: 190-8.

17.- Hankin B, Abramson L, Moffitt T, Silva P, Mcgee R, Angell $K$ : Development of depression from preadolescence to young adulthood: emerging gender differences in a 10-yera longitudinal study. J Abnorm Psychol 1998; 107: 128-40.

18.- Junta Nacional Auxilio Escolar y Becas: http:// www.junaeb.cl/focalizacion/ive.htm; 2007.

19.- Bravo M, Ribera J, Rubio-Stipec $M$, et al: Testretest reliability of the Spanish version of the Diagnostic Interview Schedule for Children (DISC-IV). Journal of Abnormal Child Psychology 2001; 29: 43344.

20.- Shaffer D, Fisher P, Lucas C, Dulcan M, Schawbstone $M$ : National Institute of Mental Health
Diagnostic Interview Schedule for Children Version IV (DISC-IV); 2000. Columbia University: New York.

21.- Canino G, Shrout P, Rubio-Stipec M, et al: The DSMIV rates of child and adolescent disorders in Puerto Rico. Arch Gen Psychiatry 2004; 61: 85-93.

22.- González Tejera G, Canino $G$, Ramírez $R$, et al: Examining minor and major depression in adolescent. Journal of Child Psychology and Psychiatry 2005; 46: 888-99.

23.- Cova F, Bravo E, Valenzuela B, Henríquez ML: Prevalencia de síntomas depresivos en adolescentes de Concepción, Revista Chilena de Pediatría 2007; 78: 151-9.

24.- Cova F: La epidemiología psiquiátrica y el debate actual respecto al concepto de trastorno mental. Revista de Psiquiatría Clínica 2006; XLIII: 31-6.

25.- Eaton Ww, Neufeld K, Chen Ls, Cai G: A comparison of self-report and clinical diagnostic interviews for depression: Diagnostic Interview Schedule for Clinical Assessment in Neuropsychiatry in Baltimore Epidemiological Catchment Area follow-up. Arch Gen Psychiatry 2000; 57: 217-22.

26.- Wing JK, Babor T, Brugha T, et al: SCAN: Schedules for clinical assessment in neuropsychiatry. Arch Gen Psychiatry 1990; 47: 589-93.

27.- Regier DA, Narrow WE: Defining clinically significant psychopathology with epidemiological date. En Helzer JE, Hudziac JJ, editors. Washington, DC: American Psychiatric Publishing Inc.; 2002, p. 19-30.

28.- Allan V, Horowitz Y, Wakefield J: The loss of sandness. Oxford University Press. New York; 2007. 